\title{
Genetic diversity of the Hungarian Gidran horse in two mitochondrial DNA markers
}

Nikolett Sziszkosz, Sándor Mihók, András Jávor, Szilvia Kusza

The Gidran is a native Hungarian horse breed that has approached extinction several times. Phylogenetic analysis of two mitochondrial markers (D-loop and cytochrome-b) was performed to determine the genetic characterization of the Gidran for the first time as well as to detect errors in the management of the Gidran stud book. Sequencing of $686 \mathrm{bp}$ of CYTB and 202 bp of the D-loop in 260 mares revealed 24 and 32 haplotypes, respectively, among 31 mare families. BLAST analysis revealed six novel CYTB and four D-loop haplotypes that have not been previously reported. The Gidran mares showed high haplotype (CYTB: $0.8735 \pm 0.011$; D-loop: $0.9136 \pm 0.008$ ) and moderate nucleotide (CYTB: $0.00472 \pm 0.00017$; D-loop: $0.02091 \pm 0.00068$ ) diversity. Of the 31 Gidran mare families, only 15 CYTB (48.4\%) and 17 D-loop (54.8\%) distinct haplotypes were formed using the two markers separately. Merged markers created 24 (77.4\%) mare families, which were in agreement with the mare families in the stud book. Our key finding was that the Gidran breed still possesses high genetic diversity despite its history. The obtained haplotypes are mostly consistent with known mare families, particularly when the two mtDNA markers were merged. Our results could facilitate conservation efforts for preserving the genetic diversity of the Gidran. 
1 Genetic diversity of the Hungarian Gidran horse in two mitochondrial DNA markers

2 Nikolett Sziszkosz ${ }^{1}$, Sándor Mihók ${ }^{1}$, András Jávor ${ }^{1}$, Szilvia Kusza $^{1 *}$

$3{ }^{1}$ Institute of Animal Science, Biotechnology and Nature Conservation, University of Debrecen,

4 H-4032 Debrecen, Böszörményi str. 138, Hungary

5 *Corresponding author:

6 email: kusza@agr.unideb.hu

7 Phone: 00-36-52-508-444

8 Fax: 00-36-52-486-285 


\section{ABSTRACT}

10 The Gidran is a native Hungarian horse breed that has approached extinction several times.

11 Phylogenetic analysis of two mitochondrial markers (D-loop and cytochrome-b) was performed

12 to determine the genetic characterization of the Gidran for the first time as well as to detect errors

13 in the management of the Gidran stud book. Sequencing of $686 \mathrm{bp}$ of CYTB and $202 \mathrm{bp}$ of the D-

14 loop in 260 mares revealed 24 and 32 haplotypes, respectively, among 31 mare families. BLAST

15 analysis revealed six novel CYTB and four D-loop haplotypes that have not been previously

16 reported. The Gidran mares showed high haplotype (CYTB: $0.8735 \pm 0.011$; D-loop: $0.9136 \pm$

17 0.008) and moderate nucleotide (CYTB: $0.00472 \pm 0.00017$; D-loop: $0.02091 \pm 0.00068)$

18 diversity. Of the 31 Gidran mare families, only 15 CYTB (48.4\%) and 17 D-loop (54.8\%) distinct haplotypes were formed using the two markers separately. Merged markers created 24 (77.4\%) mare families, which were in agreement with the mare families in the stud book. Our key finding was that the Gidran breed still possesses high genetic diversity despite its history. The obtained haplotypes are mostly consistent with known mare families, particularly when the two mtDNA markers were merged. Our results could facilitate conservation efforts for preserving the genetic

24 diversity of the Gidran.

25 KEY WORDS

26 CYTB - diversity - D-loop - Gidran - stud book error 


\section{INTRODUCTION}

Conservation of domestic animal breeds has played an ever increasing part in biodiversity preservation. Hungary realized relatively early the unique value of maintaining the genetic diversity of endangered species (Bodó 1985). The Gidran is one of the smallest horse populations among Hungarian horses (Pataki 1996). It was crossed with and influenced many other breeds in Eastern Europe and is closely associated with the Kisbér Half Breed, which is another traditional Hungarian breed. Chestnut is the usual colour of the Gidran, but other colours common to the Arab horse occur in this breed. In addition to having cultural and genetic value, the Gidran is well known for its athletic speed, agility, endurance, well-balanced temperament, and robust build (Glyn 1971). Due to its unique characteristics, the Gidran is widely used in many equestrian sports, such as horse jumping and carriage driving, and achieves outstanding results in international competitions. Its origin goes back to 1816 , but the Gidran was only registered as a separate breed in 1885 by the Austrian Ministry of Defence (Jónás et al. 2006).

During its uncertain history, two major bottleneck effects drove the breed to the edge of extinction. Fortunately, the reconstruction and regeneration of the Gidran began because of a few dedicated breeders in the 1990's. Retrenching the number of mare families was a key moment in this preservation, which took into account that each mare family has more than twenty generations of breeding history (Jónás et al. 2006). As a consequence, the final number of mare families was determined, and the latest version of the official Gidran stud book was published in 2005 (Mihok 2005). The date of the establishment of each mare families and the name of the founder mares are summarized in the Online Supporting Information (Tables S5.). Although, the ongoing restoration of the Gidran is a notable example of gene pool protection (Mihok \& Bodo 2003), the status of this horse is still endangered. According to the Domestic Animal Diversity Information System database of the Food and Agriculture Organization (FAO DAD-IS), the Hungarian Gidran population is composed of 298 mares and 21 stallions, but smaller populations also exist in Romania and Bulgaria (DAD-IS, 2014). Therefore, maintaining Gidran biodiversity is an important challenge not only for gene preservation but also from the point of view of the World Heritage (Mihok \& Bodo 2003). Within the framework of breed conservation, genetic characterization acts an important aspect of maintaining breed integrity and managing genetic resources (Glowatzki-Mullis et al. 2006). Mitochondrial DNA (mtDNA) analysis has been used in phylogenetic and domestication studies and displays a high level of genetic variation among maternal lineages in horses (Achilli 
et al. 2012; Cieslak et al. 2010; Jansen et al. 2002). Contrary to genomic DNA, mtDNA has several unique features: it consists of a single circular molecule, it follows almost exclusively maternal inheritance, and its replication occurs independently of the cell cycle. The horse mitochondrial genome is composed of approximately 16,660 nucleotides, which encode 13 polypeptides that form part of 4 protein complexes (CI, CIII, CIV and CV) of the OXPHOS system, 22 transfer RNAs, and 2 ribosomal RNA. Due to its strict maternal inheritance, individuals within a maternal family line should share the same mtDNA haplotypes, thereby allowing an evaluation of maternal line assignment accuracy (Wan et al. 2004). Several investigations have shown that using two or more mtDNA markers might be more robust and powerful for genetic diversity analysis (Pedrosa et al. 2005). Therefore, analysis of sequence variations of mtDNA such as $C Y T B$ or D-loop region is an outstanding approach for the mapping of the Gidran's maternal lineage.

The main goal of this study is to examine the genetic diversity and relations among the Gidran maternal lines. We present the first phylogenetic characterization of the Gidran for the identification of rare or distinct mtDNA haplotypes using $686 \mathrm{bp}$ and $202 \mathrm{bp}$ sequences of the mitochondrial $C Y T B$ and D-loop, respectively. The second aim of the recent study was to recognize the overlapping haplotypes or errors in the management of the stud book to gain a better understanding of the genetic variability among the Gidran mare families. Our results could complement the recent conservation strategies to maintain the genetic diversity of this traditional horse breed.

\section{MATERIALS AND METHODS}

\section{Hair sample collection and DNA extractions}

Hair samples were collected from 260 mares representing the 31 Gidran mare families (borodi 1, 2, 3, 5, 6, 7, 14, 17, 18, 19, mezöhegyesi 1, 2, 3, 4, 5, 6, 7, 8, 9, 11, 12, 13, 14, 15, 17, 18, 19, 21 and népies 9, 22, 23) in Hungary. The mare families of two horses (247G and 202G) were unknown. Total genomic DNA was extracted from the hair follicles according to the FAO protocol (FAO/IAEA, 2004). All horses in this study were client-owned on which no harmful invasive procedure was performed; and there was no animal experimentation according to the legal definitions in Europe (Subject $5 f$ of Article 1, Chapter I of the Directive 2010/63/UE of the European Parliament and of the Council).

\section{PCR amplification and sequencing}

Based on the reference Equus caballus mtDNA sequences (GenBank accession nr.: X79547 and JN398377), a 1092 bp length fragment of the cytochrome b (CYTB) gene was 
92 amplified using our own primer pairs designed with Primer3, which is free software available

93 online (Untergasser et al. 2012). The synthesized primers were as follows: $14115 F$ (5'-

94 TTCCCACGTGGAATCTAA CC-3') and 15206R (5'-ACTAACATGAATCGGCGGAC-3'). The

$95297 \mathrm{bp}$ segment of the D-loop region was amplified with previously published primer pairs

96 (Priskin et al. 2010). PCR amplifications were performed in $20 \mu \mathrm{L}$ reaction volumes comprising

$970.1 \mathrm{mM}$ dNTPs (Thermo Fisher Scientific, Waltham, MA, USA), 5x Colorless GoTaq Flexi

98 Buffer (Promega, Madison, WI, USA), $0.75 \mathrm{mM} \mathrm{MgCl}_{2}$ (Promega, Madison, WI, USA), 0.125

$99 \mathrm{mM}$ of each primer (Sigma-Aldrich, St. Louis, MO, USA), 0.75 U GoTaq DNA polymerase

100 (Promega, Madison, WI, USA), and $2 \mu \mathrm{L}(40-200 \mathrm{ng} / \mu \mathrm{L})$ horse DNA extract. Amplifications

101 were carried out with MJ Research PTC-200 Thermal Cycler (MJ Research, Watertown, MA,

102 USA). The protocol included pre-denaturation at $95{ }^{\circ} \mathrm{C}(10 \mathrm{~min})$, followed by 35 cycles of $95^{\circ} \mathrm{C}$

103 ( $30 \mathrm{sec}), 62{ }^{\circ} \mathrm{C}(45 \mathrm{sec}), 72{ }^{\circ} \mathrm{C}(30 \mathrm{sec})$, and then by a final extension at $72{ }^{\circ} \mathrm{C}$ for $10 \mathrm{~min}$ and a 4

$104{ }^{\circ} \mathrm{C}$ hold. For verification the length of the fragments, all PCR products $(5 \mu \mathrm{L})$ were examined by

105 standard agarose gel electrophoresis using 2\% agarose gel and stained with Gelred (Biotium,

106 Hayward, CA, USA. The PCR amplification were sufficient for 250 (CYTB), 246 (D-loop)

107 individuals. Finally, the PCR amplicons were purified using a DNA/RNA Extraction (PCR-M

108 Clean up System) Kit (Viogene-BioTek, Taipei, Taiwan). PCR products were commercially

109 sequenced by Macrogen Sequencing Service (Macrogen, Amsterdam, the Netherlands), PCR

110 primers were also used as sequencing primers. The obtained DNA sequences were compared with

111 the reference sequences from GenBank using Clustal X (Thompson et al. 1997).

112 Data analyses

113 Phylogenetic analysis was conducted for a total of 250 (CYTB), 246 (D-loop) individuals.

114 Combined CYTB and D-loop analysis was limited to those 242 horses where the PCR

115 amplifications were successful for both markers. BioEdit v7.2.5 (Hall 2004) sequence alignment

116 editor software was used to proof and correct individual electropherograms of the sequences. All

117 sequence alignments were performed using a general reference sequence (GenBank accession nr.:

118 X79547) and a latterly used reference sequence (GenBank accession nr.: JN398377) is also used

119 in DomeTree, wich is toolkit for mtDNA analyses in domesticated animals (Peng et al. 2015).

120 Complementary sequences were assembled and truncated to a length of $686 \mathrm{bp}(C Y T B)$ and 202

121 bp (D-loop) to allow for maximum sample size.

122 A BLAST search in the NCBI database was used to determine any previously unreported

123 haplotypes. Standard diversity measures, such as polymorphic sites (Ps), haplotype (h) and

124 nucleotide diversity (p), were calculated by DNASP 5.0 software (Rozas et al. 2003). A pairwise 
125 distance matrix between the mtDNA haplotypes was independently calculated for the $C Y T B$ and

126 D-loop by the nucleotide p-distance (Nei \& Kumar 2000). Maximum likelihood (ML) phylogeny 127 was constructed using the Hasegawa-Kishino-Yano (HKY) plus gamma (CYTB) and Tamura 3128 parameter (T92) plus gamma model (D-loop) by the inbuilt model generator in MEGA5 (Tamura

129 130 131 132 133 134 135 et al. 2011). An Equus asinus sequence (GenBank accession no.: NC001788) was used as an outgroup. Bootstrap analyses (1000 replications) were used to assess the confidence of each node. According to the polymorphic sites, both haplotypes were assigned to the DomeTree (Peng et al. 2015) and D-loop haplotypes were also clustered into the haplogroups had been defined by Jansen (2012). A phylogenetic network based on merged CYTB and D-loop regions was constructed by use of a median-joining algorithm (Bandelt et al. 1999) as implemented in the Network 4.1 program.

\section{RESULTS}

Based on the sequence comparisons of the mitochondrial CYTB and D-loop markers, the Gidran horses showed high genetic variability. Twenty-three polymorphic sites were detected in the $C Y T B$ sequences, corresponding to two indels (e.g., insertion and deletion) and 21 single nucleotide polymorphisms (SNPs) with two transversions, and representing $3.35 \%$ of the analysed DNA sequence. Within the D-loop region, 26 polymorphic sites were found (one indel and 25 SNPs with a transversion) representing $12.9 \%$ of the analysed DNA sequence (Table 1.). Both mtDNA regions were $\mathrm{A} / \mathrm{T}$ rich with the following nucleotide frequencies: $\mathrm{T}: 27.7 \%, \mathrm{C}$ : 31.5\%, A: $27.3 \%$ and G: $13.5 \%$ in $C Y T B$ and T: $30.8 \%$, C: $24.7 \%, \mathrm{~A}: 33.3 \%$ and G: $11.2 \%$ in Dloop. The A and T content was richer (55\% and 64.1\%) in both the CYTB and D-loop regions, respectively. These data were in accordance with the order of nucleotide composition in the vertebrate mitochondrial genome.

The calculated haplotype diversity (h) of the $C Y T B$ and D-loop markers was $0.8735 \pm$ 0.011 and $0.9136 \pm 0.008$, whereas the nucleotide diversity was $0.00472 \pm 0.00017$ and 0.02091 \pm 0.00068 , respectively. The paired genetic distances between the haplotypes were $0.001-0.013$ $(C Y T B)$ and 0.005-0.063 (D-loop). All phylogenetic analyses were performed for both separate and combined mtDNA markers; a summary of the calculated genetic diversity parameters and the haplotypes of the Gidran mares are presented in Table 1.

Analysis of $686 \mathrm{bp}$ of the $C Y T B$ and $202 \mathrm{bp}$ of the D-loop regions revealed 24 and 32 distinct haplotypes among the 31 Gidran mare families, the sequences of the CYTB and D-loop haplotypes are available in GenBank database under accession no.: KT792934 - KT792957 and KT818891 - KT818922. The haplotypes differ from the reference sequences (GenBank accession 
nr.: X79547 and JN398377) by a maximum of 6 (CYTB) and 9 (D-loop) nucleotides. The polymorphic sites of both mtDNA markers are summarized in the Online Supporting Information (Table S1 \& Table S2). Ht1 Сутв $(n=54), H t 2_{\text {Сутв }}(n=49)$ and Ht6 ${ }_{\text {Сутв }}(n=44)$ were the three most frequent haplotypes of the $24 C Y T B$ haplotypes, whereas seven $\left(\mathrm{Ht} 11_{\mathrm{CY}}, \mathrm{Ht} 17_{\mathrm{CYTB}}, \mathrm{Ht} 18_{\mathrm{CYTB}}\right.$, $\mathrm{Ht} 20_{\text {Сутв }}, \mathrm{Ht} 21_{\mathrm{CY} B}, \mathrm{Ht} 22_{\text {СутB }}$, and $\mathrm{Ht} 24_{\mathrm{CY}}$ ) were limited to only a single mare. The maximum likelihood tree representing the phylogenetic relationship among the $24 C Y T B$ haplotypes of the 250 Gidran mares is presented in Figure 1. In the case of the D-loop haplotypes, Ht6 $6_{\text {D-loop }}(n=47)$,

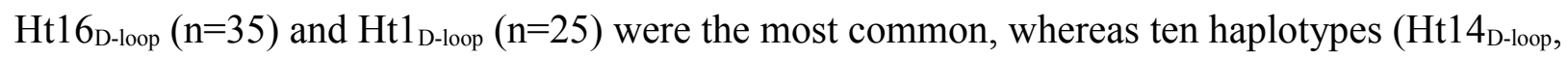

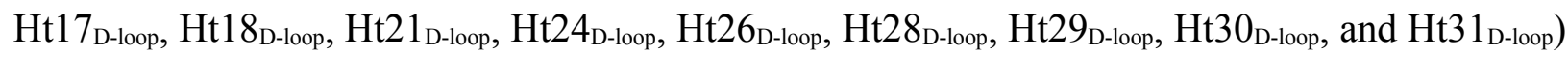
were singletons. The BLAST search revealed six CYTB $\left(\mathrm{Ht} 5_{\mathrm{CYTB}}, \mathrm{Ht} 8_{\mathrm{CYTB}}, \mathrm{Ht} 11_{\mathrm{CYTB}}, \mathrm{Ht} 14_{\mathrm{CYTB}}\right.$,

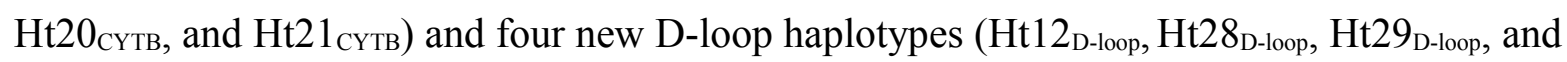
Ht32 ${ }_{\text {D-loop}}$ ), which have not been published in NCBI database so far, and summarized in the Online Supporting Information (Table S1 \& Table S2). The combined CYTB and D-loop haplotypes could be clustered into ten (A, A1a, AB, B1, B1a, H, H-I, JK, M-N, and M-Q) haplogroups according to the DomeTree (Figure S1.) (Peng et al. 2015). Furthermore, D-loop haplotypes could also be assigned to seven of the major D-loop haplogroups defined by Jansen et al. (2002) with the following haplogroup distribution: A: 31\%, B: 3\%, C: 28\%, D: 19\%, E: 3\%, F: 13\%, and G: 3\% (Figure 2). Among the 18 haplogroups (A-R) reported by Achilli et al. (2012), haplogroup E was not present in the Gidran native horses considered (Table S3 \& Table S4). Our additional key objectives were to identify errors in the Gidran stud book and to test the efficiency of the mtDNA markers for the separation of the mare families by haplotypes. Of the 31 mare families, $15(48.4 \%)$ and 17 (54.8\%) formed unique haplotypes according to the CYTB and D-loop markers, respectively. Three mare families (borodi 14 and 18 and mezöhegyesi 1) could be separated exclusively by the $C Y T B$, whereas four mare families (mezöhegyesi 2, 3, 19 and borodi 2) could be detected using only D-loop as a marker. Interestingly, individuals in the borodi 1 and 7 mare families formed a common haplotype when the two mtDNA markers were combined. On the other hand, seven mare families (mezőhegyesi 7, 8, and 21; borodi 5, 17, and 19; and népies 23) could be separated by the combination of CYTB and D-loop markers. Five mare families (mezöhegyesi 5, 11, 13, and 14 and borodi 18) could not be isolated with either of the markers. A median-joining network based on the combination of CYTB and D-loop regions was constructed by Network 4.1 and is shown in Figure 3.

\section{DISCUSSION}


The Gidran is a rare and endangered native Hungarian horse breed; therefore, the

191

192

193

194

195

196

197

198

199

200

201

202

203

204

205

206

207

208

development of an effective conservation strategy is extremely urgent. A determination of phylogenetic relationships and the verification of stud book accuracy could be the first steps in the maintenance of the genetic pool (Bodó et al. 2005). To date, contrary to the situation with the Hucul, which is another, but well-studied native Hungarian horse breed (Czerneková et al. 2013; Georgescu et al. 2011; Kusza et al. 2013), no data are available on the genetic structure and diversity of the Gidran. In this regard, through DNA sequence comparisons of CYTB and the Dloop, we investigated matrilineal diversity for Gidran horses in Hungary. Our research presents the first molecular phylogenetic study of the Gidran and covers approximately $87 \%$ (260/298) of the Hungarian Gidran mares from 31 traditional maternal lineages. Our mtDNA based data would tend to make the recent conservation strategies more successful to prevent genetic erosion in the Gidran.

First, we analysed the $C Y T B$ and D-loop mitochondrial DNA markers of the Gidran mares. According to our results, both markers reflected a strong genetic variety in Gidran. The presence of 24 and 32 mtDNA haplotypes with a total of $3.35 \%$ and $12.9 \%$ polymorphic sites in the $C Y T B$ and D-loop fragments reflect the broad genetic base of the Gidran maternal lines. Similar results have been found in the Hucul or Zemaitukai breeds which have relatively high nucleotide diversity despite suffering from severe bottlenecks during their histories (Cothran et al. 2005; Kusza et al. 2013).

The observed 32 D-loop haplotypes is similar in number to that reported for the Lusitano (27 haplotypes/145 horses) (Lopes et al. 2005), Lipizzan (37 haplotypes/212 horses) (Kavar et al. 2002) Arabian (27 haplotypes/200 horses) (Bowling et al. 2000) and higher than in Kiso (7 haplotypes/ 136 horses) (Takasu et al. 2014). The calculated D-loop haplotype and nucleotide diversities are inconsistent with earlier horse mtDNA studies. The calculated nucleotide diversity was $0.02091 \pm 0.00068$, which is quite similar to the Iranian horse population $(0.02 \pm 0.000)$ reported by (Moridi et al. 2013). This relatively high number indicates, that the Gidran is genetically more diverse than, for example, the Kerry Bog $(0.0155 \pm 0.0040)$ and Sulphur Mustang breeds $(0.001 \pm 0.002)$, but not more diverse than the Marwari $(0.03973 \pm 0.01262)$ or Sorraia breeds (0.104 \pm 0.012) (Devi \& Ghosh 2013; Luís et al. 2006; Prystupa et al. 2012). Data from the $C Y T B$ sequences also confirmed the abundant genetic diversity of the Gidran. In the case of $C Y T B$, the nucleotide diversity was lower in comparison to the D-loop, but it is similar to that observed in Chinese domestic horses, where the nucleotide diversity was between 0.00488 and 0.00186 while haplotype diversity was between 0.706 and 0.975 (Yue et al., 2011). Quin et 
223

224

225

226

227

228

229

230

231

232

233

234

235

236

237

al. (2009) sequenced a $1140 \mathrm{bp}$ length $C Y T B$ region in 22 Lichuan horses and also obtained high haplotype diversity and nucleotide diversity values ( 0.840 and 0.048 , respectively). In a study, where $C Y T B$ was used for the characterization of maternal genetic origins and diversity of 323 horses from 13 Chinese indigenous breeds and 84 reference sequences from GenBank, 114 haplotypes were identified (Yue et al. 2012). The observed high D-loop and CYTB haplotype diversity confirm the multiple maternal origin of the Gidran, which might be explained by the fact that the recently known maternal lines were established by 16 founder mares (Mihok 2005). These data suggest that although the Gidran is one of the smallest Hungarian horse population, the genetic diversity of the maternal lineage is preserved (Takasu et al. 2014).

BLAST showed that almost all the haplotypes found in the Gidran samples are identical to other domestic horse haplotypes in GenBank except for six CYTB and five D-loop haplotypes that have not been described for any other horse breed yet. Because uncommon haplotypes have an increased risk of extinction (Lopes et al. 2005), these distinct haplotypes support the importance of maintaining rare individuals and also emphasize the genetic diversity of the Gidran.

Pedigree analysis also plays a key role in breeding programmes, which aim to maintain genetic diversity of endangered populations (Bokor et al. 2013). Our further aim was to recognize overlapping haplotypes among mare families and thereby detect incidental errors in the Gidran stud book. Using the mtDNA markers alone was not sufficiently effective because neither $C Y T B$ nor D-loop sequences made the alignment of the haplotypes to each of the mare families reasonably acceptable. To improve the efficiency of our data, starting from the study of Pedrosa et al. (2005), a phylogenetic analysis with the combined CYTB and D-loop markers was also performed (Figure 3). Although, the results showed several inconsistencies in the distribution of the 49 common $C Y T B$ and D-loop haplotypes within the 31 mare families, we found this approach more effective to screen for registry errors rather than using only one mtDNA marker. Altogether, pedigree records were problematic in seven mares $(2.89 \%)$ registered in the Gidran stud book. This number is small compared with Lipizzan and Polish horses where the discrepancies were $11 \%$ between pedigree data and mtDNA haplotypes in studies on both breeds (Głażewska et al. 2007; Kavar et al. 2002). The data collected in the present study indicates the management of the Gidran stud book was appropriate over the years. The seven problematic mares belonged to a different cluster (independent of the DNA markers and their combination) than suggested according to the Gidran stud book. Several reasons could explain the described inconsistencies. However, errors may have been made in the management of the stud book 
256 because the approximately 200 years of existence of the maternal line is a very short time for the

257 formation of a distinct haplotype of each mare family (Devi \& Ghosh 2013).

258 Four horses did not possess their own cluster. Among them, two animals from the borodi

2597 mare family formed a common cluster with borodi 1. Furthermore, two individuals of the

260 mezőhegyesi 5 mare family shared a haplotype with animals of mezőhegyesi 11 . These

261 discrepancies are in concordance with the mare family's history (Mihok 2005). Individuals of

262 borodi 1 and 7 shared the same haplotype. Possibly, mare families sharing the same haplotypes

263 belong to the same mare family, which is, as a consequence of the incomplete pedigree data, now

264 split into different mare families (Kavar et al. 2002). In contrast, based on the analysis of the two

265 mitochondrial markers separately and combined, individuals of the mezöhegyesi 4 mare family

266 formed two distinct haplotypes. This observation was confirmed by the available historical data,

267 which suggests that the mezőhegyesi 4 mare family diverged over the years (Mihok 2005).

\section{Conclusions}

269 In addition to providing our first insight into the maternal mitochondrial diversity in the 270 rare native Hungarian Gidran breed, this study also provided the opportunity to compare

271 molecular genetic results with stud book data. Our key finding was that high matrilineal diversity 272 was observed in the Gidran breed using both $C Y T B$ and D-loop markers despite the history of the 273 breed. Moreover, the obtained haplotypes are mostly consistent with stud book's mare families, 274 particularly when the two mtDNA markers were combined. Gidran breeders are recommended to 275 take this information into account in the future. 


\section{REFERENCES}

Achilli A, Olivieri A, Soares P, Lancioni H, Kashani BH, Perego UA, Nergadze SG, Carossa V, Santagostino M, and Capomaccio S. 2012. Mitochondrial genomes from modern horses reveal the major haplogroups that underwent domestication. Proceedings of the National Academy of Sciences 109:2449-2454.

Bandelt H-J, Forster P, and Röhl A. 1999. Median-joining networks for inferring intraspecific phylogenies. Molecular biology and evolution 16:37-48.

Bodó I. 1985. Hungarian activities on the conservation of domestic animal genetic resources. Animal Genetic Resources Information 4:19-25.

Bodó I, Alderson L, and Langlois B. 2005. Conservation genetics of endangered horse breeds: Wageningen Academic Pub.

Bokor A, Jónás D, Ducro B, Nagy I, Bokor J, and Szabari M. 2013. Pedigree analysis of the Hungarian Thoroughbred population. Livestock Science 151:1-10.

Bowling A, Del Valle A, and Bowling M. 2000. A pedigree-based study of mitochondrial d-loop DNA sequence variation among Arabian horses. Animal genetics 31:1-7.

Cieslak M, Pruvost M, Benecke N, Hofreiter M, Morales A, Reissmann M, and Ludwig A. 2010. Origin and history of mitochondrial DNA lineages in domestic horses. PLoS One 5:e15311.

Cothran E, Juras R, and Macijauskiene V. 2005. Mitochondrial DNA D-loop sequence variation among 5 maternal lines of the Zemaitukai horse breed. Genetics and Molecular Biology 28:677-681.

Czerneková V, Kott T, and Majzlík I. 2013. Mitochondrial D-loop sequence variation among Hucul horse. Czech J Anim Sci 58:437-442.

DAD-IS. 2014. Domestic Animal Diversity Information System database of the FAO. Available at: http://www.fao.org/dadis/

Devi KM, and Ghosh SK. 2013. Molecular phylogeny of Indian horse breeds with special reference to Manipuri pony based on mitochondrial D-loop. Molecular biology reports 40:5861-5867.

FAO/IAEA Agriculture Biotechnology Laboratory. 2004. Handbook of Laboratory Exercises, IAEA Laboratories, Seibersdorf, Austria

Georgescu S, Manea M, Dudu A, and Costache M. 2011. Phylogenetic relationships of the Hucul horse from Romania inferred from mitochondrial D-loop variation. Genet Mol Res 10:4104-4113.

Głażewska I, Wysocka A, Gralak B, Prus R, and Sell J. 2007. A new view on dam lines in Polish Arabian horses based on mtDNA analysis. Genetics, Selection, Evolution : GSE 39:609-619. 10.1186/12979686-39-5-609

Glowatzki-Mullis M, Muntwyler J, Pfister W, Marti E, Rieder S, Poncet P, and Gaillard C. 2006. Genetic diversity among horse populations with a special focus on the Franches-Montagnes breed. Animal Genetics 37:33-39.

Glyn R. 1971. The world's finest horses and ponies: George G. Harrap \& Co. Ltd.

Hall T. 2004. BioEdit version 7.0. 0. Distributed by the author, website: www mbio ncsu edu/BioEdit/bioedit html.

Hasegawa M, Kishino H, and Yano T-a. 1985. Dating of the human-ape splitting by a molecular clock of mitochondrial DNA. Journal of molecular evolution 22:160-174.

Jansen T, Forster P, Levine MA, Oelke H, Hurles M, Renfrew C, Weber J, and Olek K. 2002. Mitochondrial DNA and the origins of the domestic horse. Proceedings of the National Academy of Sciences 99:10905-10910. 
319

320

321

322

323

324

325

326

327

328

329

330

331

332

333

334

335

336

337

338

339

340

341

342

343

344

345

346

347

348

349

350

351

352

353

354

355

356

357

358

359

360

361

362

363

364

365

366

367
Jónás S, Hajba N, Mihók S, and Vörös J. 2006. Monograph of the Gidran horse. . Debrecen, Hungary: Center-Print Press.

Kavar T, Brem G, Habe F, Sölkner J, and Dovč P. 2002. History of Lipizzan horse maternal lines as revealed by mtDNA analysis. Genetics Selection Evolution 34:635-648.

Kusza S, Priskin K, Ivankovic A, Jedrzejewska B, Podgorski T, Jávor A, and Mihók S. 2013. Genetic characterization and population bottleneck in the Hucul horse based on microsatellite and mitochondrial data. Biological Journal of the Linnean Society 109:54-65.

Lopes MS, Mendonca D, Cymbron T, Valera M, da Costa-Ferreira J, and Machado Ada C. 2005. The Lusitano horse maternal lineage based on mitochondrial D-loop sequence variation. Anim Genet 36:196-202. 10.1111/j.1365-2052.2005.01279.x

Luís C, Bastos-Silveira C, Cothran EG, and do Mar Oom M. 2006. Iberian origins of New World horse breeds. Journal of Heredity 97:107-113.

Mihók S. 2005. Gidran Studbook. Budapest: Kisberi and Gidran Horse Breeders Association.

Mihók S, and Bodó I. 2003. Utilisation of animal genetic resources in Hungary 1. Hungarian Agricultural Research (Hungary).

Moridi M, Masoudi A, Vaez Torshizi R, and Hill E. 2013. Mitochondrial DNA D-loop sequence variation in maternal lineages of Iranian native horses. Animal Genetics 44:209-213.

Nei M, and Kumar S. 2000. Molecular evolution and phylogenetics: Oxford University Press.

Pataki B. 1996. Questions of preservation of the Gidran horse breed. Allattenyesztes es Takarmanyozas (Hungary).

Pedrosa S, Uzun M, Arranz J-J, Gutiérrez-Gil B, San Primitivo F, and Bayón Y. 2005. Evidence of three maternal lineages in near eastern sheep supporting multiple domestication events. Proceedings of the Royal Society B: Biological Sciences 272:2211-2217.

Peng MS, Fan L, Shi NN, Ning T, Yao YG, Murphy RW, Wang WZ, and Zhang YP. 2015. DomeTree: a canonical toolkit for mitochondrial DNA analyses in domesticated animals. Molecular ecology resources.

Priskin K, Szabó K, Tömöry G, Bogácsi-Szabó E, Csányi B, Eördögh R, Downes C, and Raskó I. 2010. Mitochondrial sequence variation in ancient horses from the Carpathian Basin and possible modern relatives. Genetica 138:211-218.

Prystupa J, Juras R, Cothran E, Buchanan F, and Plante Y. 2012. Genetic diversity and admixture among Canadian, Mountain and Moorland and Nordic pony populations. animal 6:19-30.

Rozas J, Sánchez-DelBarrio JC, Messeguer X, and Rozas R. 2003. DnaSP, DNA polymorphism analyses by the coalescent and other methods. Bioinformatics 19:2496-2497.

Takasu M, Ishihara N, Tozaki T, Kakoi H, Maeda M, and Mukoyama H. 2014. Genetic Diversity of Maternal Lineage in the Endangered Kiso Horse Based on Polymorphism of the Mitochondrial DNA D-Loop Region. The Journal of Veterinary Medical Science 76:1451.

Tamura K, Peterson D, Peterson N, Stecher G, Nei M, and Kumar S. 2011. MEGA5: molecular evolutionary genetics analysis using maximum likelihood, evolutionary distance, and maximum parsimony methods. Molecular biology and evolution 28:2731-2739.

Thompson JD, Gibson TJ, Plewniak F, Jeanmougin F, and Higgins DG. 1997. The CLUSTAL_X windows interface: flexible strategies for multiple sequence alignment aided by quality analysis tools. Nucleic acids research 25:4876-4882.

Untergasser A, Cutcutache I, Koressaar T, Ye J, Faircloth BC, Remm M, and Rozen SG. 2012. Primer3-new capabilities and interfaces. Nucleic acids research 40:e115-e115.

Wan QH, Wu H, Fujihara T, and Fang SG. 2004. Which genetic marker for which conservation genetics issue? Electrophoresis 25:2165-2176.

Yue XP, Qin F, Campana MG, Liu DH, Mao CC, Wang XB, Lan XY, Chen H, and Lei CZ. 2012. Characterization of cytochrome b diversity in Chinese domestic horses. Animal Genetics 43:624-626.

10.1111/j.1365-2052.2011.02298.x 
368 TABLE

369 Table 1

370 Summary of the diversity parameters and haplotypes found in the Gidran mares according

371 to the mtDNA $C Y T B$ and D-loop sequences and their combination

$\begin{array}{cccccccc} & \text { Number } & \text { Number } & \text { Number of } & \text { Number of } & & & \\ \text { mtDNA } & \text { of } & \text { of } & \text { detected } & \text { new } & \text { Polymorphi } & \text { Haplotype } & \text { Nucleotide } \\ \text { marker } & \text { nucleotid } & \text { hoplotypes } & \text { c sites } & \text { diversity } \pm \text { SD } & \text { diversity } \pm \text { SD } \\ & \text { es } & \text { animals } & \text { haplotypes } & & & & \end{array}$

$C Y T B$

686

250

24

6

23

$0.8735 \pm 0.011$

$0.00472 \pm$

0.00017

D-loop 202

246

32

26

$0.9136 \pm 0.008$

$0.02091 \pm$ 0.00068

$\begin{array}{crrrrrrr}\text { CYTB \& } & 893 & 242 & 49 & - & 49 & 0.9402 \pm 0.006 & 0.00837 \pm \\ \text { D-loop } & & & & & & 0.00020\end{array}$

\section{$372 \quad(\mathrm{SD}=$ Standard Deviation $)$}


373

374

375

376

377

378

\section{LEGENDS TO FIGURES}

Figure 1

A maximum likelihood tree represents the phylogenetic relationship among the 24 haplotypes based on 686 bp of protein coding mtDNA CYTB of 250 Gidran mares. The phylogenetic tree was based on the Hasegawa-Kishino-Yano (HKY) model of evolution with gamma distribution of rates and 1000 bootstrap replicates (Hasegawa et al. 1985). The polymorphic sites considered relative to the X79547 reference sequence. Asterisks represent the new haplotypes.

\section{Figure 2}

A maximum likelihood tree represents the phylogenetic relationship among 32 haplotypes based on 202 bp of mtDNA of the D-loop region of 246 Gidran mares. The phylogenetic tree was based on the Tamura 3-parameter (T92) model of evolution with gamma distribution of rates and 1000 bootstrap replicates (Tamura et al. 2011). Haplotypes were clustered into seven main haplogroups (A-F) described by Jansen et al. (2002). The polymorphic sites considered relative to the X79547 reference sequence. Asterisks represent the new haplotypes.

\section{Figure 3}

Median-joining network based on combined nucleotide sequences (892 bp) of the mitochondrial CYTB and D-loop of 242 mares representing the 31 Gidran mare families. Circle size is proportional to the sequence frequency and the colours represent different mare families. The number of transverse bars on the branches represent the number of nucleotide substitutions between the nodes. In the cases of $247 \mathrm{G}$ and $202 \mathrm{G}$ (yellow), the mare families are unknown. The abbreviations of the Gidran mare families are the following: $m=$ mezöhegyesi; $\mathrm{b}=$ borodi; and $\mathrm{n}=$ népies. 


\section{FIGURES}

397 Figure 1

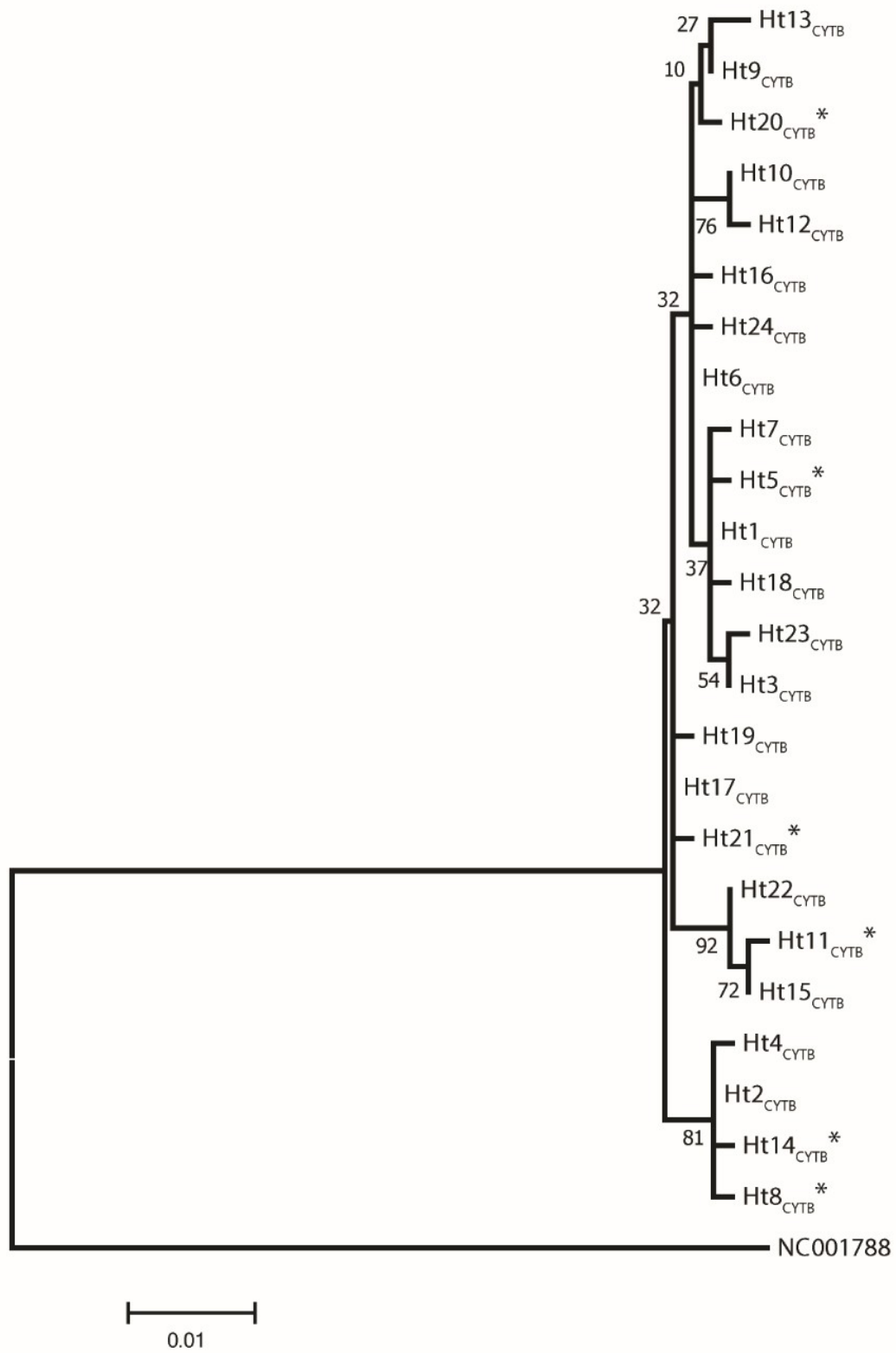




\section{Figure 2}

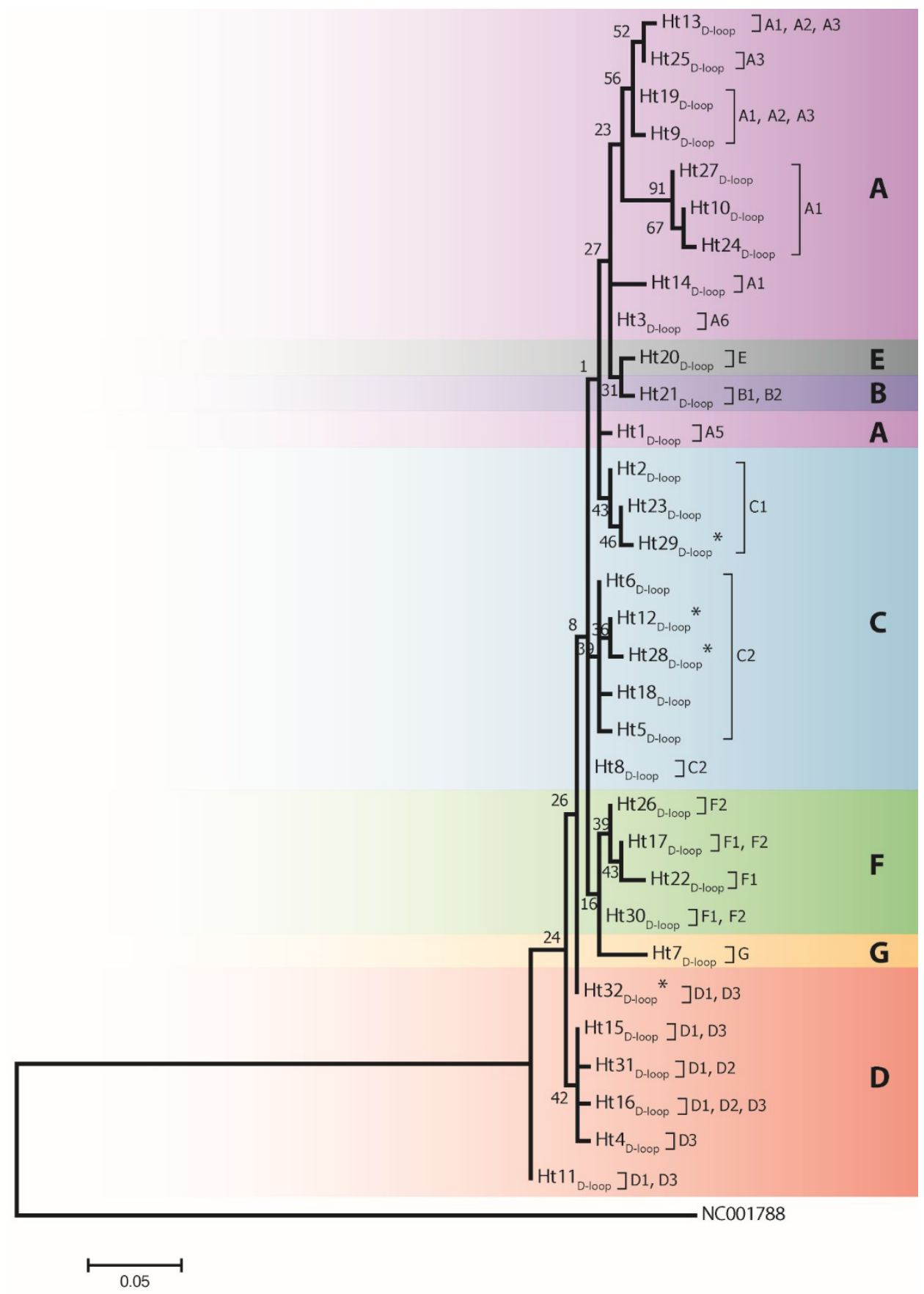


Figure 3

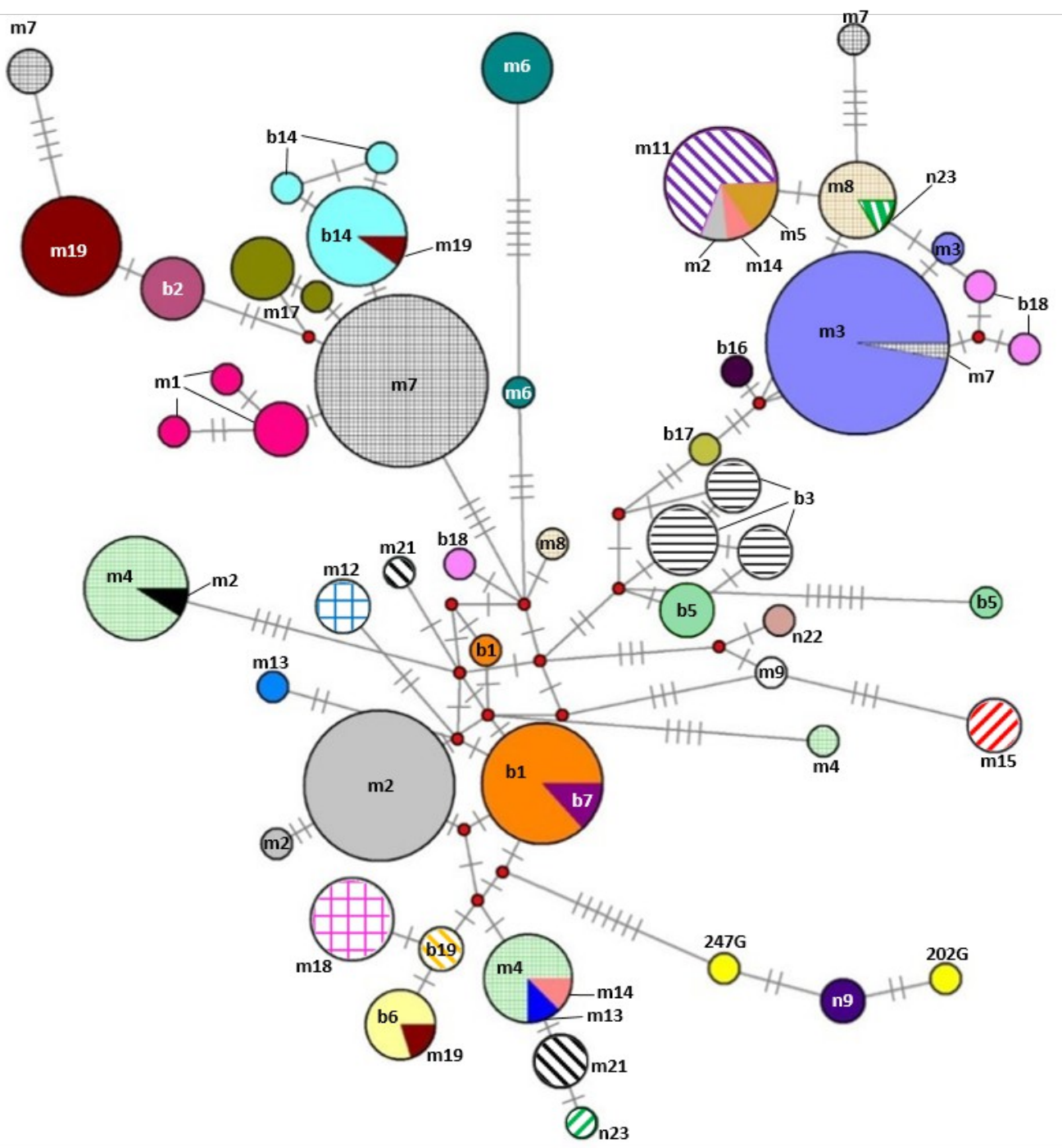


400 SUPPORTING INFORMATION

401 Additional Supporting Information may be found in the online version of this article:

402 Table S1. Variable site positions in 686 bp long mtDNA CYTB sequence of 24 Gidran haplotypes.

403 New haplotypes are emphasized by gray colour.

404 Table S2. Variable site positions in 202 bp of mtDNA D-loop region of 32 Gidran haplotypes.

405 New haplotypes are emphasized by gray colour.

406 Table S3. The nominations of the detected CYTB haplotypes in different horse studies.

407 (*Unpublished data; **Haplotypes were clustered into haplogroups)

408 Table S4. The nominations of the detected D-loop haplotypes in different horse studies.

409 (*Unpublished data; **Haplotypes were clustered into haplogroups)

410 Table S5. Summary of the 32 mare families according to the Gidran studbook.

411 Figure S1. Classification tree of combined CYTB and D-loop haplotypes of Gidran mares

412 according to the DomeTree. The polymorphic sites considered relative to the JN398377 reference

413 sequence, which is identical with Haplotype 1 (H1). 